\title{
Promoter sequences driving inducible gene expression in human T- and NK-cells
}

\author{
S. Kulemzin ${ }^{*}$, D. Matvienko ${ }^{1,2}$, A. Sabirov ${ }^{1,2}$, A. Sokratyann ${ }^{1,2}$, D. Chernikova ${ }^{1,2}$, \\ T. Belovezhets ${ }^{1,2}$, A. Chikaev ${ }^{1}$, A. Taranin ${ }^{1,2}$, A. Gorchakov ${ }^{1,2}$ \\ ${ }^{1}$ Institute of Molecular and Cellular Biology SB RAS, Novosibirsk, Russia \\ ${ }^{2}$ Novosibirsk State University, Novosibirsk, Russia \\ *e-mail:skulemzin@mcb.nsc.ru
}

Key words: chimeric antigen receptor, cancer, reporter, inducible promoter, NK cell lines

Motivation and Aim: Cytotoxic activity of T- and NK-cells can be efficiently retargeted against cancer cells using chimeric antigen receptors (CARs) and rTCRs. In the context of solid cancers, use of armored CAR T- and NK cells secreting additional anticancer molecules such as cytokines, chemokines, antibodies, BiTEs, inverted cytokine receptors, and checkpoint inhibitors, appears particularly promising, as this may help overcome immunosuppressive tumor microenvironment, attract bystander immune cells and boost CAR T/NK-cell persistence. Placing the expression of such molecules under the transcriptional control downstream of CAR-mediated T/NK-cell activation offers the advantage of targeted delivery, high local concentration, and reduced toxicity. Several canonic DNA sequences that are known to function as activation-inducible promoters in human $\mathrm{T}$ and $\mathrm{B}$ cells have been described to date and typically encompass the multimers of NFkB and NFAT binding sites. However, relatively little is known about the DNA sequences that may function as activation-driven switches in the context of NK cells. We set out to compare the functionality of several activation-inducible promoters in primary human T cells, as well as in NK cell lines NK-92 and YT.

Methods and Algorithms: To this end, lentiviral constructs were engineered to express two fluorescent reporters: mCherry under NFATx4, NFkBx2, NFkBx5, NFkBx10, NFkBx20, as well as two variants of CD69 promoter and copGFP under the strong constitutive promoter of the human EF1a gene. Reporters lacking the inducible promoter or driven by the weak constitutive PGK promoter served as the controls. Pseudotyped lentiviral particles obtained using these constructs were transduced into primary human $\mathrm{T}$ cells and NK-92 and YT cell lines expressing a CAR specific for PSMA.

Results: The transgenic cells obtained were activated by conventional TCR cross-linking (T cells) or via a CAR (CAR-NK cell lines). Promoter activity before and after activation was assayed using FACS analysis. In T cells, the CD69 promoter encompassing CNS1 and CNS2 regions displayed the highest signal/noise ratio. Intriguingly, in the context of CAR-YT cell line neither of the seven promoters tested displayed acceptable activation profile. In CAR-NK-92 cells, the largest fold activation (which was modest) was achieved with the NFkBx20 promoter, however its expression was clearly leaky in "resting" nonactivated cells.

Conclusion: Unlike in $\mathrm{T}$ cells, the robust activation-driven inducible expression of genetic cassettes in NK cells requires unbiased identification of promoter sequences. Acknowledgements: Supported by the RSF (16-14-10237) and the Basic scientific research program project (0310-2018-0012). 